\title{
Erratum: Sex hormones establish a reserve pool of adult muscle stem cells
}

\author{
Ji-Hoon Kim, Gi-Chan Han, Ji-Yun Seo, Inkuk Park, Wookjin Park, Hyun-Woo Jeong, Su Hyeon Lee, \\ Sung-hwan Bae, Jinwoo Seong, Min-Kyu Yum, Sang-Hyeon Hann, Young-Guen Kwon, Daekwan Seo, \\ Man Ho Choi and Young-Yun Kong
}

Nature Cell Biology 18, 930-940 (2016); published online 22 August 2016; corrected after print 8 September 2016

In the version of this Article originally published, in the key in Fig. 1b, the red and black data points were incorrectly labelled; the red data points should have been labelled 'DHT' and the black data points as 'Veh'. Furthermore, in the key in Fig. 2c, the red and black data points were also incorrectly labelled; the red data points should have been labelled ' $\mathrm{N} 1^{\Delta \mathrm{SC}}$ ', and the black data points as ' $\mathrm{N} 1^{\text {WT' }}$. These errors have been corrected in the online versions of the Article. 